\title{
JammJoint: A Variable Stiffness Device Based on Granular Jamming for Wearable Joint Support
}

\author{
Simon Hauser, Student Member, IEEE, Matthew Robertson, Auke Ijspeert, Member, IEEE, \\ and Jamie Paik, Member, IEEE
}

\begin{abstract}
In robotics, controlling the stiffness of the joints that contribute to the robots' degree of freedom dictates the adaptability, versatility, and safety of the whole system. We can achieve variable stiffness or impedance in a robotic system purely by the control or by introducing new material or mechanisms to address cases that require innate safety through system compliancy. This paper presents JammJoint, a compliant and flexible wearable robot, which uses jamming of granular media to vary its stiffness. It consists of a silicone sleeve with hollow sections that are filled with cubic rubber granules and subjected to different levels of vacuum pressure. Unlike contemporary vacuum-based actuators or systems, JammJoint is wearable, portable, and autonomous: It uses a powerful miniature vacuum pump, a small battery, and bluetoothenabled electronics. Experiments revolving around bending and torsional stiffness show that the system is able to achieve up to a fourfold increase in spring stiffness. Further measurements of individual variable stiffness structures indicate that for other modes of deformation, including simply supported bending or compression for alternative linear applications, higher changes in stiffness over a factor of seven are possible. These aspects make mobile jammingbased stiffness variation as wearable joint assistance promising for future applications such as rehabilitation after injuries and joint support in challenging working conditions.
\end{abstract}

Index Terms-Wearable robots, soft material robotics, variable stiffness joint, vacuum jamming, hydraulic/pneumatic actuators.

\section{INTRODUCTION}

I $\mathrm{N}$ THE case where the energy efficiency is a crucial design feature of a robot or a robot is required to safely interact with humans, new compliant actuator designs are better suited than classical, stiff robot designs which typically excel in pick-and-place applications. Such compliant actuators have the ability to reduce impact forces, store and release energy

Manuscript received September 17, 2016; accepted January 2, 2017. Date of publication January 18, 2017; date of current version February 7, 2017. This paper was recommended for publication by Associate Editor X. Liu and Editor Y. Sun upon evaluation of the reviewers' comments. This work was supported by the Swiss National Science Foundation under Projects 153299 and 163292.

S. Hauser and A. Ijspeert are with the Biorobotics Laboratory, School of Engineering, Institute of Bioengineering, Ecole Polytechnique Fédérale de Lausanne, Lausanne 1015, Switzerland (e-mail: simon.hauser@epfl.ch; auke.ijspeert@epfl.ch).

M. Robertson and J. Paik are with the Reconfigurable Robotics Laboratory, School of Engineering, Institute of Mechanical Engineering, Ecole Polytechnique Fédérale de Lausanne, Lausanne 1015, Switzerland (e-mail: matthew.robertson@epfl.ch; jamie.paik@epfl.ch).

This letter has supplementary downloadable material available at http:// ieeexplore.ieee.org.

Color versions of one or more of the figures in this letter are available online at http://ieeexplore.ieee.org.

Digital Object Identifier 10.1109/LRA.2017.2655109
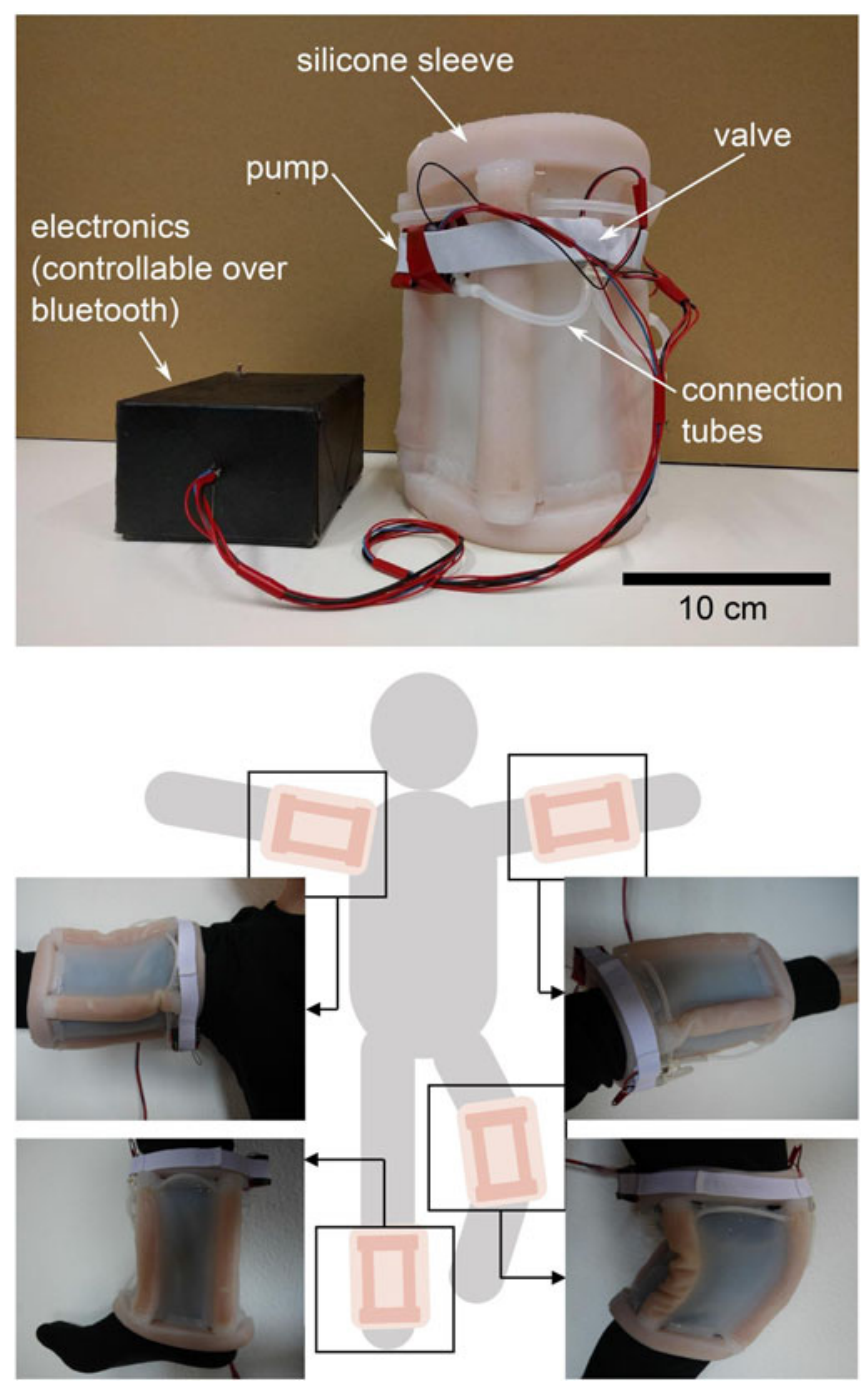

Fig. 1. The JammJoint device. Top: the electronic box on the left contains a microcontroller, bluetooth module and battery. A miniature pump, small-scale valves and connection tubes are mounted directly on the silicone sleeve. The sleeve with pump and valves weights $700 \mathrm{~g}$, the electronic box is $250 \mathrm{~g}$ of which the battery weights $100 \mathrm{~g}$. Bottom: application examples of the JammJoint device on different human joints.

through their compliance, and add safety. Thus, these actuators are the preferred choice in various applications such as rehabilitation, prostheses, manipulation, bio-inspired robotics and wearable robotics [1]. However, the compliance in the system often needs to be well-tuned to a specific task. If a robot is 
to perform a variety of tasks, a corresponding prescription of different compliance settings is required, giving rise to variable stiffness mechanisms.

The design of variable stiffness manipulators and joints is an active field and many prototypes have been developed in the past. Some of them use pretensioning of a spring to stiffen a joint [2].

For highly articulated robots (e.g. [3]), the stiffness usually cannot be changed unless a compliant element is integrated in the design [4]. This essentially represents a series elastic actuator (SEA) which has the ability to adjust its stiffness by changing the elastic element [5]. For continuum robots [6], tightening tendons [7] and Shape Memory Polymers (SMP) [8] are used to vary the stiffness. Magneto-rheological dampers can adjust their damping properties which in turn also has an effect on their spring stiffness (e.g. [9]). This can be also considered as a variable stiffness joint, although rather as a side effect.

Recently, the field of granular jamming is receiving more attention since on the one hand it can be used as a continuum robot and on the other hand it offers state switching, i.e. a transition from a soft to a hard state. In an open area, a granular medium (e.g. sand) behaves fluid-like (continuum) where the granules can freely slide around each other, thus being soft and adaptable. By confining the space around the granules, the interparticle friction increases drastically, transforming the pack of granules into a solid-like state, resulting in a hard structure [10]. The material and shape of the granules has found to play a key role in the mechanical properties of a filled membrane. Likewise, the material of the membrane influences the behavior. A comparison between membrane materials and different types of granules can be found in e.g. [11] and the concept has been investigated for a number of applications such as grippers [12], [13], dampers [14], [15] and actuators [16], [17].

In [18], a proposition of a variable stiffness joint based on granular jamming can be found. The usual hard granules used for jamming are replaced with compliant granules. This resulted in a vacuum-dependent stiffness of the joint. In their experiments, a finger-like membrane filled with different types of compliant granules has been tested for its ability to increase the bending stiffness depending on the pressure level inside the membrane. Their idea was to fix an array of such columns around a joint and vary the stiffness by controlling the pressure in these columns. The joint can either be active (actuated by inflating the columns) or passive (the columns only vary their stiffness but do not provide actuation).

This concept was the inspiration for creating the proposed JammJoint which is designed to support and stabilize human joints during post-injury rehabilitation or for daily assistance of chronic biomechanical impairment. Similar to existing orthotic devices for this purpose, this device provides constraints to joint motion in selected directions to reduce unwanted loading and motion, while allowing free or low impedance motion in other directions for maintaining principle joint functionality. In general, most existing devices are only uni-axial, providing support and restriction in all but one direction, or they offer only fixed or manually adjustable levels of supporting stiffness. The JammJoint enables automated tuning of both the direction
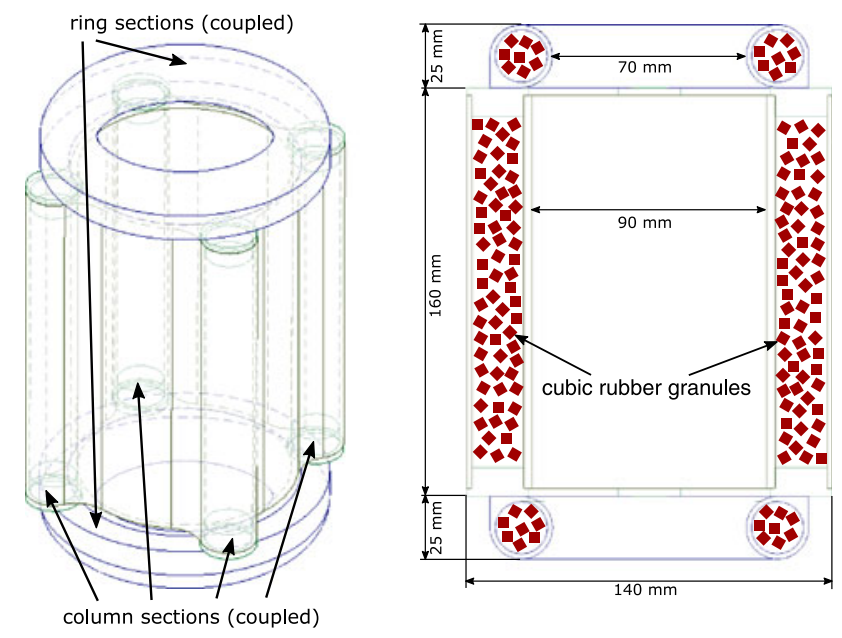

Fig. 2. Silicone sleeve. Left: The complete sleeve. Two hollow rings (blue; coupled to share the same pressure) are attached at each end of a cylinder with four integrated, hollow columns (green; coupled to share the same pressure). Right: Cross-section of the sleeve. The hollow sections in the rings and columns are filled with cubic rubber granules.

and magnitude of joint axis support by applying distributed, independently controlled variable stiffness elements in parallel with the biological joint. The variable stiffness is enabled by vacuum induced jamming of compliant granules, as previously investigated. The novelty of the design is that the structural part of the device doubles as the enclosing membrane to enable the jamming, which also directly facilitates its wearability around a joint. Additionally, in contrast to other applications of granular jamming where a large, powerful vacuum pump is required externally, JammJoint uses a miniature vacuum pump directly integrated in the device. This makes the system unique in the field of granular jamming as it is as a whole wearable and - adding a battery to power the electronics - portable and autonomous.

\section{Description of the JammJoint Device}

The JammJoint device consists of several parts: the sleeve with its jammable columns and fixation rings, the pump together with the valves and tubes, the electronics controlling the system and the user interface on a bluetooth enabled smartphone. The complete device can be seen in Fig. 1 (except for the smartphone) and each separate part is described in detail below.

\section{A. Silicone Sleeve}

The sleeve is made out of highly stretchable silicone (Ecoflex 00-30) and consists of three parts. The main section is formed by a cylindrical shape with four evenly distributed hollow columns integrated along the cylinder and a total height of $210 \mathrm{~mm}$. These columns are coupled together by tubes and share the same pressure. The diameter of the hollow cavity in the columns is $20 \mathrm{~mm}$. On each side of the cylinder, hollow rings are attached, likewise with a hollow cavity of $20 \mathrm{~mm}$ and coupled together. Both rings and the hollow sections of the cylinder are filled with cubic rubber granules with a side length of $4 \mathrm{~mm}$ (Neukadur ProtoFlex HS 75). Schematics of the sleeve are depicted in 


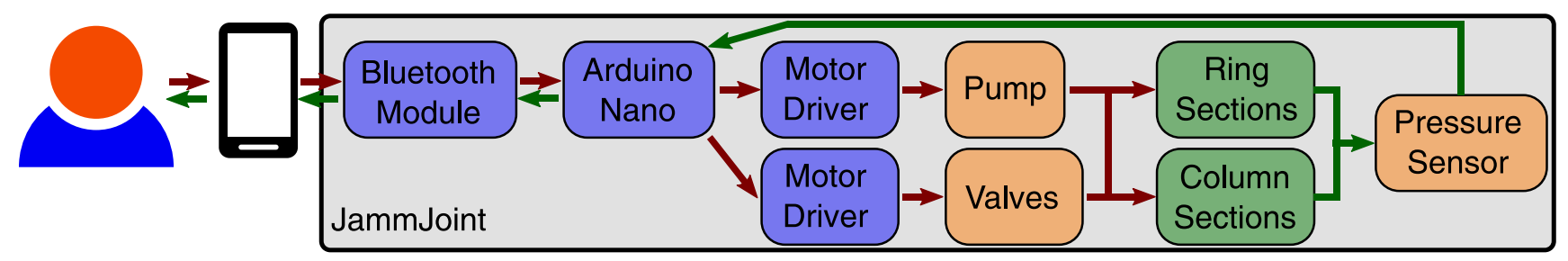

Fig. 3. Schematics of the full JammJoint system. A user (on the left) inputs the desired commands into a smartphone which communicates with a bluetooth module attached to the Arduino microcontroller. It controls the pump and valves over motor drivers, changing the pressure level inside the ring and column sections. The pressure is measured by a sensor and fed back to the microcontroller which also shares this information with the user over the smartphone interface.

Fig. 2. The figure displays the active controllable $2 \mathrm{DoF}$ of the system: 2 rings and 4 columns are independently controlled.

\section{B. Pump and Valves}

A miniature pump (Schwarzer SP 100 EC-DU, serial configuration) creates a vacuum up to -800 mbar versus atmospheric pressure inside the rings and columns (200 mbar absolute pressure). The airflow of the pump is controlled with three miniature solenoid valves (SMC S070C-SAG-32) and the level of vacuum is measured with a single pressure sensor (Honeywell 015PAAA5).

\section{Electronics and Bluetooth Interface}

A microcontroller (Arduino Nano V3.0) controlls the pump, valves and pressure sensor. Additionally, a bluetooth module (JY-MCU) is attached to the microcontroller, allowing a bluetooth serial communication. The electronics are powered by a 12 V LiPo battery (Conrad energy BEC 11.1 V 1300 mAh 12 C). A bluetooth enabled smartphone can establish a connection to the bluetooth module. For this work, the free app BlueTerm $2^{1}$ has been used. The device is controlled with a few simple commands: "r" and "c" direct the airflow either to the rings or columns, the numbers $1-8$ are used to set the pressure in the respective section from 900 mbar to 200 mbar absolute pressure and the number 0 sets a section to atmospheric pressure. The complete system can be seen in Fig. 3 where the smartphone is the only external hardware; JammJoint is fully autonomous otherwise.

\section{EXPERIMENTS}

\section{A. Full-Device Characterization}

The objective of the experimental measurement of the complete device is to obtain the range of its stiffness variability. Since the device is designed for the use on human joints, these joints are approximated by balljoints for the purpose of this investigation. A balljoint has 3 degrees of freedom: 2 rotations around the joint in 2 perpendicular planes (further called "bending") and 1 rotation around the main axis of the joint (further called "torsion"). Whereas the torsion movement is symmetric in case of the device, bending is directional, depending on the orientation of the bending plane to the columns. For these experiments, only bending where the bending plane is in between

\footnotetext{
${ }_{1}^{1}$ available for Android in Google Play store.
}
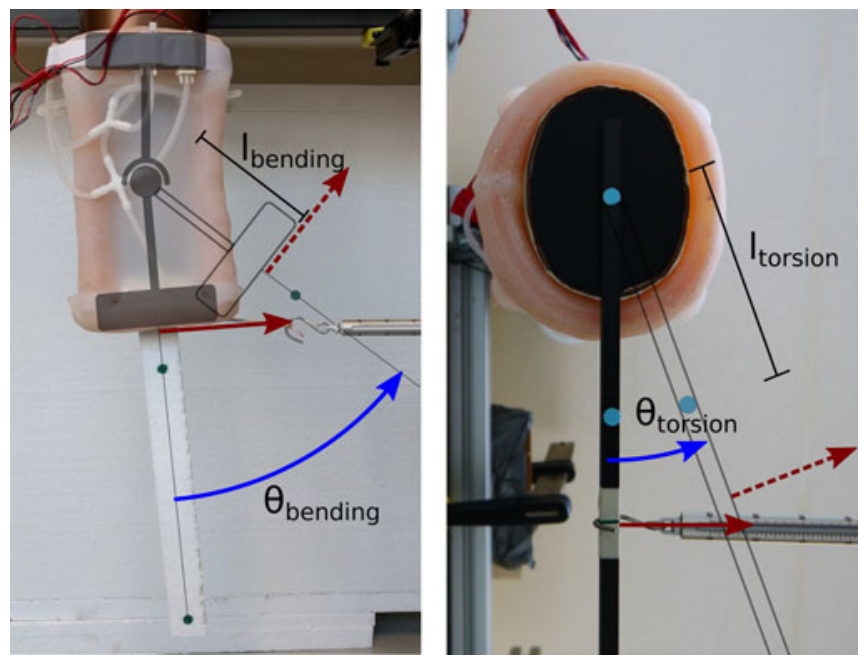

Fig. 4. Experimental setups. The red arrows show the direction of the force at resting position; the silhouette and the red dashed arrows show the orientation of the lever and the direction of the force at the rotation angle $\theta_{\text {ben din }} /$ tor sion (blue arrow). Left: Side view of the bending setup. The mechanics of the balljoint is overlaid in gray. Right: Bottom view of the torsion setup.

two columns is analyzed (see Fig. 4). This is expected to more evenly distribute the bending torques as each column is engaged in a similar way, to simplify both quantitative and qualitative results. The case where the bending axis coincides with a column is not studied as one column would receive a large compressive force and one column a large strain respectively compared to the columns located outside the bending plane. This type of loading is likely to yield unique stiffness characteristics, including enhanced effects of buckling, however this is considered to be only important for a full quantification of the device stiffness, beyond the intention of this study.

It is important to note that in a balljoint, all possible movements can be superposed. This is also achievable with the device. The two rings only serve as the way of fixing the device on the respective joint through passive adaptation and solidifying. Only the level of vacuum in the columns determines the final stiffness, therefore coupling both bending and torsional stiffness. The experimental procedure for the experiments is defined as follows: (i) fit the device around the joint, (ii) pair the JammJoint with a smartphone, (iii) solidify the rings by setting the pressure inside the rings to the lowest value (200 mbar absolute pressure), (iv) increase the level of vacuum inside the columns in steps of 100 mbar from atmospheric pressure (approx. 1000 mbar ab- 
solute pressure) to 200 mbar absolute pressure by selecting the desired pressure on the smartphone and (v) measure the torque for different angle of rotations. Fig. 4 shows the experimental setup. A camera takes pictures of the device and a force meter. Two colored dots allow the calculation of the rotation angle through image analysis and the force meter provides the applied torque, given the length of the lever.

1) Bending Torque: For measuring the bending torque, the device is put over a balljoint where torsion is restricted. The two rings are both fit over circular disks, each with the height of the ring $(25 \mathrm{~mm})$. The joint is located exactly in the middle of the device, resulting in a lever length $l_{\text {bending }}$ of $105 \mathrm{~mm}$. The upper part of the joint is fixed to a table. Each configuration is tested 3 times. A force is applied perpendicular to the lower part of the joint until the rotation angle reaches approximately $45 \mathrm{deg}$. The force then is slowly released back to zero to also measure a potential hysteresis in the rotation angle, caused by the displacement of granules. This leads to a different resting angle because the displacement of granules generates plastic deformation of the device. After every test, the columns therefore are set to atmospheric pressure to allow to manually "reset" the position of the granules inside the columns. Although this is only considering the most ideal loading case and does not represent real operation conditions where a reset would not take place (and thus hysteresis effects would occur), preventing the rearrangement of granules will be further discussed in Section V. Effects caused by the viscoelacity of the silicone have been neglected as the response time of silicone is roughly between $50 \mathrm{~ms}$ and $200 \mathrm{~ms}$ [19] which is assumed to be one order of magnitude faster than the expected movement of a human joint. The results of this series are normalized to the initial resting angle of the respective test (meaning that initially, zero applied force is zero rotation angle).

2) Torsion Torque: For measuring the torsion torque, the balljoint is removed, keeping only the upper circular disk. To avoid slipping, the upper part of the device is additionally secured and the lower circular disk is replaced by an ellipsoid. This change is assumed to have only a minor influence on the measurements. The ellipsoid has a lever attached (lever length $l_{\text {torsion }}$ is $150 \mathrm{~mm}$ ) where the force is applied in a rotational manner, twisting the device. The number of trials (3) and measurement methods are kept the same as for the previous experiments.

\section{B. Sub-Component Characterization}

Additionally, experimental measurements are taken to individually characterize the change in stiffness of the subcomponents of the wearable device, namely the rings, and the columns. A three point bending setup is used to apply a linear force at the center of a single column segment simply supported at the ends, while the applied force is recorded through a displacement range and different pressures. While the force and displacement measurements can be used to estimate the true angular bending stiffness of a column following analytic beam bending models, only the linear transverse stiffness of the column as directly tested is reported to illustrate the qualitative effect of pressure variation on column stiffness. Similarly to
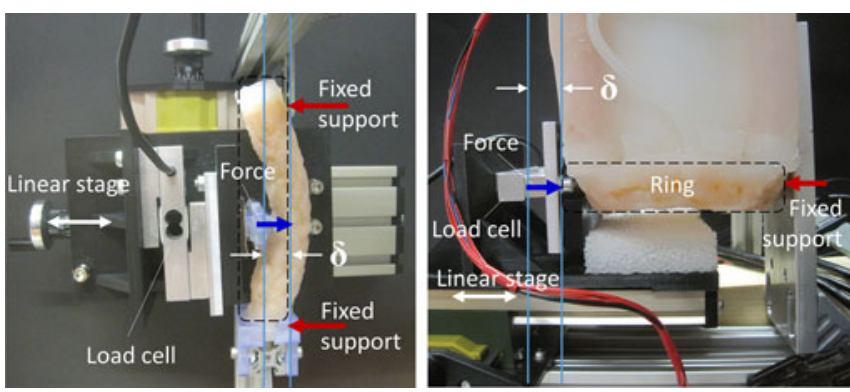

Fig. 5. Sub-component experimental characterization. A 3-point bending setup is used on the left to apply a force at the centerpoint of a single variable stiffness column. A single-axis load cell records the force applied to the column. On the right, the same apparatus is reconfigured to apply a force to the variable stiffness ring segment. In both experiments a linear stage is manually advanced and the deflection, $\delta$, is recorded at 2 millimeter intervals from 0-20 mm, equal to the width or diameter of a single column.

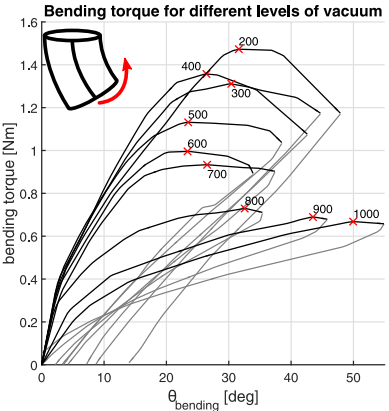

(a)

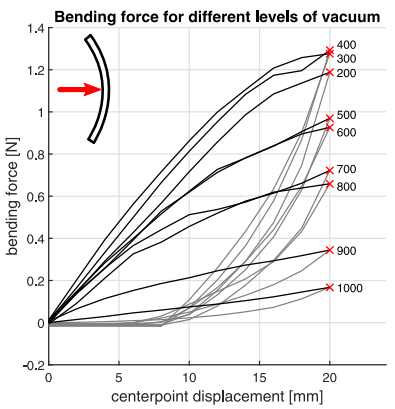

(c)

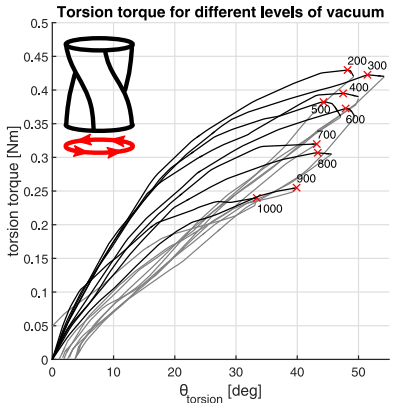

(b)

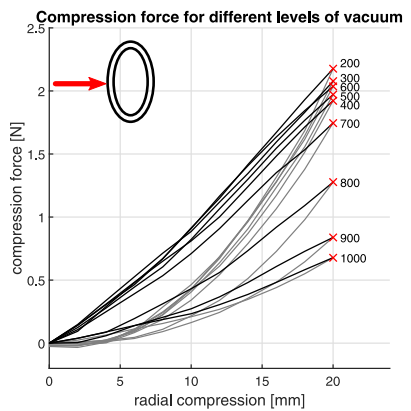

(d)
Fig. 6. Characterization experiments. Red cross: maximum torque per curve with the level of vacuum in mbar; Solid: measurements during stretching; Gray: measurements during relaxation. In all experiments, increasing the level of vacuum results in an increased reaction torque/force. (a) Bending angle versus bending torque; buckling and hysteresis effect observable. (b) Torsion angle versus torsion torque. (c) Force versus column deflection; hysteresis effect observable. (d) Force versus ring deflection.

the column measurements, the stiffness profile of a single ring segment is measured across a range of displacements and pressures. The compressive force applied across the ring is used to calculate the overall radial stiffness of the structure. The setup for each characterization test can be seen in Fig. 5 .

\section{RESUltS AND Discussion}

\section{A. JammJoint Performance in Bending}

Fig. 6(a) shows the results of the experiments performed in the bending series. Each curve is calculated with the interpolated average of the three trials per vacuum level and for each 
curve, the stretching phase (solid), relaxation phase (gray) and the maximum torque (red cross) is indicated. From these curves, the increase of the torque needed for a certain rotation angle is clearly visible. However, especially in higher vacuum, a buckling of the columns under compression can be observed, leading to a decrease of torque with larger angles. The buckling also plastically deforms the columns by rearranging the position of granules which changes the loading conditions for the relaxation phase. This can be seen from the increase in resting angles after relaxation where a hysteresis of several degrees starts to form. As the resting angle gets shifted, the respective torque at certain deflection values is lower in the relaxation phase compared to the stretching phase. Resetting the granules after each trial made sure that this hysteresis would not transfer into the next trial.

\section{B. JammJoint Performance in Torsion}

In Fig. 6(b), the results of the torsion experiments are shown. Similar to Fig. 6(a), the interpolated average is shown for stretching (solid) and relaxation phase (gray) together with the maximum torque (red crosses). The increase of the required torque is not as pronounced as in case of bending but nevertheless observable. In contrast to the previous experiments, no buckling occurs and a hysteresis in resting angles is less noticeable.

\section{Sub-Components of JammJoint}

The results from characterization of a single column at different pressures is shown in Fig. 6(c). Each curve shown represents the average of three separate trials taken for each pressure. The curves show a nearly linear stiffness relationship during stretching with a maximum force at the maximum deflection. There is also a large hysteresis observed resulting from plastic deformation at high vacuum pressures.

The stiffness profile generated from the ring sub-component measurements can be seen in Fig. 6(d). The radial stiffness of the ring can be seen to be very nearly linear, with minimal plastic deformation at any pressure, and hence minimal hysteresis upon unloading.

\section{Change of Spring Stiffness}

The results of Fig. 6(a) and (b) are summarized in Fig. 7 where the change of the respective spring stiffness is shown. For each curve in the previous experiments, the maxima (red crosses) are connected to the origin with a straight line. The slope of each line can be regarded as a hypothetical linear spring stiffness with $D_{\text {bending/torsion }}=\Delta$ torque $/ \Delta$ angle where $\Delta$ torque is the maximal torque in Nm, $\Delta$ angle the angle in deg corresponding to the maximal torque and $D_{\text {bending/torsion }}$ the spring stiffness coefficient in Nm/deg. Fig. 7 shows these spring stiffnesses for bending and torsion for the different levels of vacuum with the error bars calculated from the standard deviation of the interpolated average at the maxima. For bending, the increase in the spring stiffness is significant: the experiments show an almost fourfold increase (from $0.0134 \mathrm{Nm} / \mathrm{deg}$ to $0.0514 \mathrm{Nm} / \mathrm{deg}$ ) with the maximum stiffness value being reached at 400 mbar. For torsion, the difference is much smaller with an increase

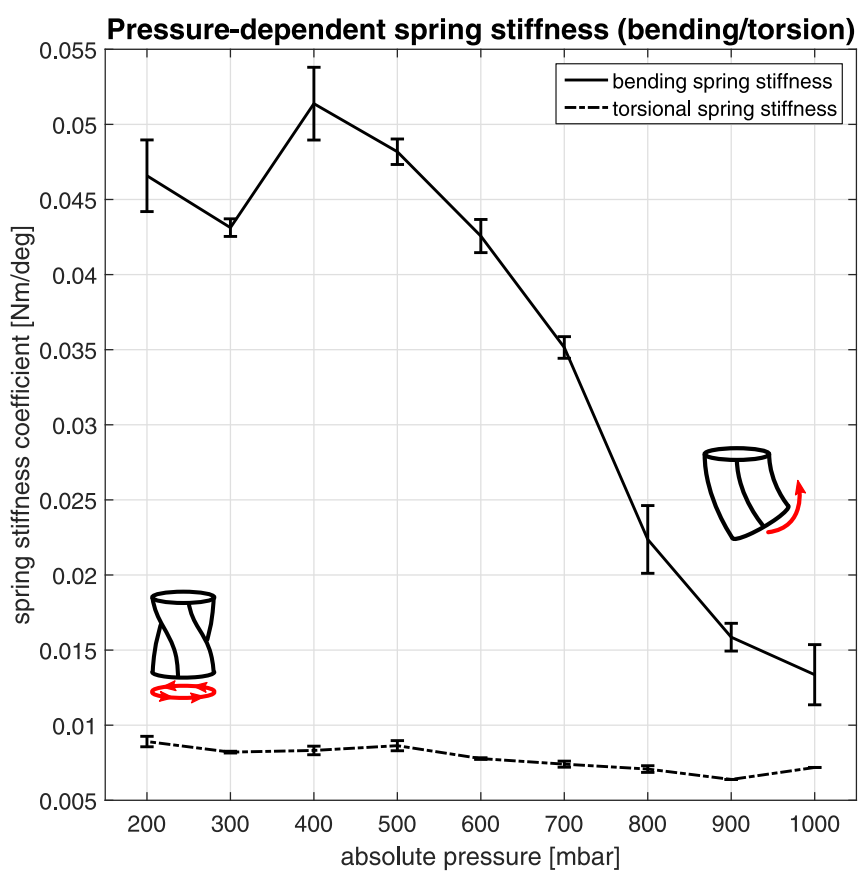

Fig. 7. Spring stiffness increase depending on the level of vacuum for bending and torsion. The values of the curves are calculated by approximating the spring behavior of each experiment with a linear spring stiffness coefficient from the origin to the maximal torque from Fig. 6(a) and (b)

of torsional spring stiffness of $40 \%$ (from $0.0064 \mathrm{Nm} / \mathrm{deg}$ to $0.0089 \mathrm{Nm} / \mathrm{deg}$ ). Even though these results are obtained by simple linear approximations of the nonlinear spring stiffness coefficients where the true "instantaneous" stiffness is angledependent, the goal of this study was not to precisely define the stiffness (possibly with the help of models) but rather to give an overview of what qualitative overall stiffness change the system can achieve.

Likewise, the results of Fig. 6(c) and(d) are summarized in Fig. 8. For each curve in the respective experiments, the maxima (red crosses) are again connected to the origin with a straight line. The slope of each line is obtained as $D_{\text {column/ring }}=$ $\Delta$ force/ $\Delta$ deflection where $\Delta$ force is the maximal force in $\mathrm{N}$, $\Delta$ deflection the distance in $\mathrm{mm}$ corresponding to the maximal force and $D_{\text {column/ring }}$ the spring stiffness coefficient in $\mathrm{N} / \mathrm{mm}$. Error bars are calculated from the standard deviation of three measurements taken at each pressure level. Both trends for the column and ring show a significant change in stiffness for different pressures. The ring stiffness increases more than threefold from 0.0339 to $0.1088 \mathrm{~N} / \mathrm{mm}$ following a pressure change from 1000 to 200 mbar. Over the same pressure range, the column stiffness changes from 0.0084 to $0.0646 \mathrm{~N} / \mathrm{mm}$; the maximum stiffness value is again observed at an intermediate pressure of 400 mbar. The maximum range indicates that an increase of stiffness over a factor of 7 is possible.

Ideally, these changes in spring stiffness are linked to a change in intrinsic material properties. In the case of jamming, the applied level of pressure suggests that the pack of granules changes its Young's modulus which is closely related to the overall spring behavior. However, modeling the effect of jamming on the Young's modulus has not been investigated as such char- 


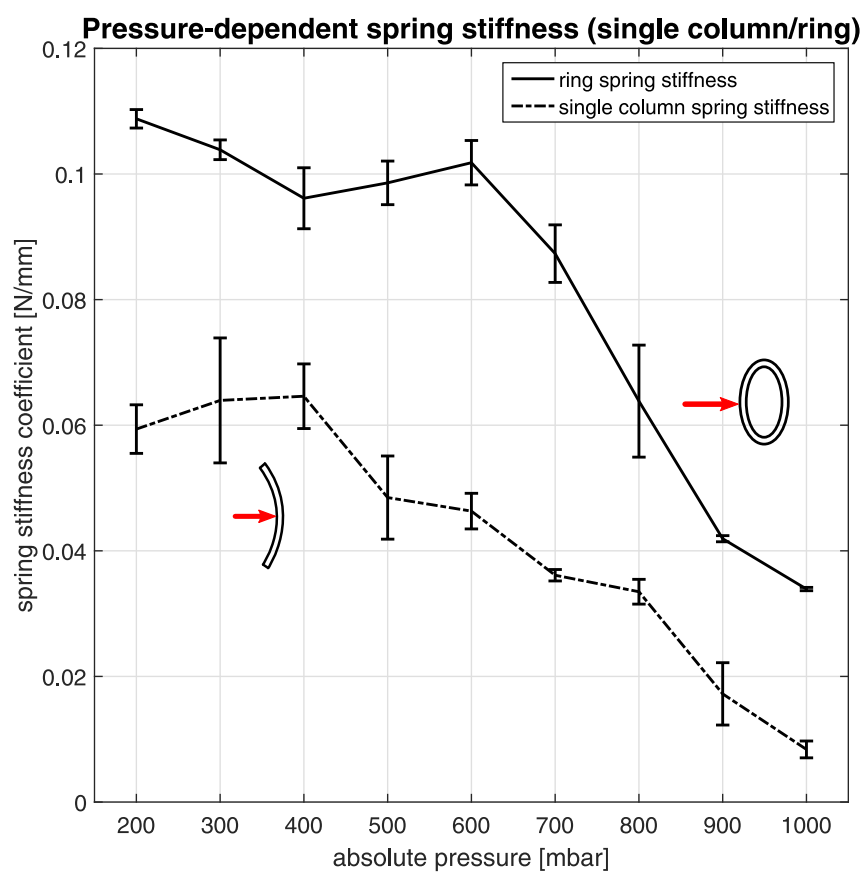

Fig. 8. Spring stiffness increase depending on the level of vacuum for a single column and ring sub-component. The values of the curves are calculated by approximating the spring behavior of each experiment with a linear spring stiffness coefficient from the origin to the maximal force from Fig. 6(c) and (d).

acterizations usually require unidirectional loading of the substrate (typically compression and tension). The performed experiments were not specifically designed for such cases. Therefore, instead of a change in intrinsic material properties, only the simple comparison of these changes to a linear spring stiffness are presented here. It is subject of ongoing research to model inter-particle reactions and define, which experiments qualify for material property characterizations in different loading scenarios.

\section{E. Additional Modes}

Firstly, besides a coupled bending-torsion-mode, an additional bending-only mode is available. This can be achieved by fitting one ring around the base of a joint and only loosely fitting the second ring (by enlarging it to a slightly bigger diameter than the other joint base). This results in the device being held in place by the fit ring but leaving the torsion rotation of the joint unrestricted. The bending stiffness still can be adjusted in the same manner as in the normal mode.

Secondly, the initial orientation of the device can be freely chosen and does not need to be known beforehand. For the purpose of the performed experiments, the initial orientation was chosen to be straight. However, with the rings solidified but the columns under atmospheric pressure, the joint can easily be moved into any desired orientation where it can be fixed by solidifying the columns. This process is reversible and can be repeated whenever required.

\section{F. Passive Adaptability, Safety and Operation Time}

The soft silicone of the sleeve and the nature of the granules allow the JammJoint to passively adapt to the wearers anatomy.
Moreover, the rings and cylinder can easily be stretched to twice their circumference, making the device applicable on different joints. This also counts for joint shapes which are not circular where the rings conform around. Once fit, after solidifying the rings to attach the device to the joint, the rings keep their conformed shape without further power. Additionally, thanks to the soft silicone, the solidified rings and columns still possess a certain softness, allowing a certain deformation of the joint e.g. through moving muscles or blood flow. The examples in Fig. 1 depict the application of the device on elbow, shoulder, ankle and knee joint.

Since the device - except the miniature pump and valves - is entirely made out of soft materials, the risk of injury for the user is greatly diminished. Moreover, since the functionality of the device relies on successfully maintaining a vacuum, puncturing any part of the silicone sleeve simply results in a slow inflation of the section to atmospheric pressure. The device therefore loses its functionality without harming a potential user (as opposing to e.g. a technology based on inflation where puncturing a membrane might result in explosion of the section). These properties are promising for the usage of such a device as joint support.

Concerning the operation time, most of the battery power is consumed when the pump is evacuating. Even though the evacuation time is somewhat slow (roughly 20 seconds) as a result of the usage of a miniature vacuum pump with low airflow, it has been assumed that the application of a joint assistance does not require a fast change in stiffness. In contrary, the advantage of the pump to be powerful but portable was regarded as being more important.

It is important to notice that a set pressure level in the sections is held without power by the off-position of the valves (assuming the sections are airtight; the current device inflates at around 25 mbar per minute due to sealing imperfections). Thus, the operation time depends almost only on the number of evacuation cycles the pump is able to perform with one battery charge. All testing and experiments were performed with one single charge of the used LiPo-battery, performing roughly 150 evacuation cycles without completely discharging the battery.

\section{CONCLUSION}

In this article, a joint assistance device based on the jamming of granular media is presented. Cubic rubber granules are filled into two separate hollow sections of a silicone sleeve, rings and columns. These sections can independently be evacuated to a desired level of vacuum by a powerful miniature vacuum pump directly integrated in the device. A small LiPo-battery powers the electronics and the microcontroller with an attached bluetooth module. This enables the system to communicate with e.g. a bluetooth enabled smartphone, making it portable and autonomous.

The level of vacuum inside the sections varies the stiffness property of the device, measured for two different modes: bending stiffness and torsional stiffness. Decreasing the pressure from 1000 mbar to 200 mbar absolute pressure results in an almost fourfold increase in bending stiffness and a $40 \%$ increase in torsional stiffness. Additionally, besides the normal mode of a coupled bending-torsional stiffness change, a bending-only 
mode is available. Individually, the subsections of the wearable device are shown to have high versatility with a large range of stiffness adjustment available (up to seven times stiffer) for alternate deformation modes, including simply supported bending and radial compression. Further, given by the method of jamming granular media, the device can be moved in any orientation when the sections are under atmospheric pressure and fixed in this orientation by solidifying (evacuating) the sections. The pressure level then can be kept without additional power. At last, the highly stretchable silicone in combination with rubber granules creates a safe to use, adaptable, versatile device which can be applied on different joint sizes and shapes.

For future work, different directions could be followed. The mechanical design of the sleeve could be changed to include more columns to make their distribution more uniform for bending. If required, the columns could be separated from each other to control the vacuum in each column independently (contrary to the current design where all the columns are connected together to share the same pressure). Although this would require more valves, the independent control of multiple columns in parallel would also increase the robustness and reliability of the total system [20]. Designing custom electronics would allow for a full integration into the sleeve, eliminating the need for additional cables. A different design to increase the torsional stiffness could be tested. Alternate mechanical configurations with different combinations of sub-component elements (columns and rings) might also be used to create entirely new forms of a variable stiffness wearable device. Further, the materials used for the sleeve and granules can be subject to future studies. While the soft silicone enables the jamming of the granules, it also adds much softness to the system even when the sections are under the highest vacuum. If necessary, a harder material or a different combination of materials could further increase the overall stiffness and the achievable change in both bending and torsional stiffness. Although it has not been tested here, the method of jamming does not interfere with actuation through inflation. Thus, with the integration of additional valves only, the same system can be used to provide active actuation. The design and choice of materials might change if inflation is also considered. One issue in the current design is that if the columns are inflated, the granules collect on the bottom of the enlarged cavity, making further jamming impossible which relies on uniformly distributed granules. A way of preventing such an undesirable rearrangement in the enlarged volume has to be developed. This could also enable automatic resetting of the granules (e.g. under atmospheric pressure) to diminish hysteresis effects, as described in Section III. Lastly, further experiments are needed to provide the basis to model changes in intrinsic material properties such as Young's modulus.

\section{REFERENCES}

[1] R. Ham, T. Sugar, B. Vanderborght, K. Hollander, and D. Lefeber, "Compliant actuator designs," IEEE Robot. Autom. Mag., vol. 16, no. 3, pp. 81-94, Sep. 2009.

[2] K. Melo, M. Garabini, G. Grioli, M. Catalano, L. Malagia, and A. Bicchi, "Open source VSA-CubeBots for rapid soft robot prototyping," in Proc. Robot Makers Workshop Conjunction Robot. Sci. Syst., 2014, pp. $1-5$.

[3] J. Shang et al., "An articulated universal joint based flexible access robot for minimally invasive surgery," in Proc. 2011 IEEE Int. Conf. Robot. Autom., 2011, pp. 1147-1152.

[4] M. Vespignani, K. Melo, S. Bonardi, and A. J. Ijspeert, "Role of compliance on the locomotion of a reconfigurable modular snake robot," in Proc. 2015 IEEE/RSJ Int. Conf. Intell. Robots Syst., 2015, pp. 2238-2245.

[5] D. Rollinson et al., "Design and architecture of a series elastic snake robot," in Proc. Intell. Robots Syst., 2014, pp. 4630-4636.

[6] G. Robinson and J. B. C. Davies, "Continuum robots-A state of the art," in Proc. 1999 IEEE Int. Conf. Robot. Autom., 1999, vol. 4, pp. 2849-2854.

[7] U. Çulha and F. Iida, "Enhancement of finger motion range with compliant anthropomorphic joint design," Bioinspiration Biomimetics, vol. 11, no. 2, Feb. 2016, Art. no. 026001.

[8] A. Firouzeh, M. Salerno, and J. Paik, "Soft pneumatic actuator with adjustable stiffness layers for multi-DoF actuation," in Proc. 2015 IEEE/RSJ Int. Conf. Intell. Robots Syst,. 2015, pp. 1117-1124.

[9] D. Guo and H. Hu, "Nonlinear stiffness of a magneto-rheological damper," Nonlinear Dyn., vol. 40, pp. 241-249, 2005.

[10] A. J. Liu and S. R. Nagel, "Nonlinear dynamics: Jamming is not just cool any more," Nature, vol. 396, no. 6706, pp. 21-22, 1998.

[11] A. Jiang, G. Xynogalas, P. Dasgupta, K. Althoefer, and T. Nanayakkara, "Design of a variable stiffness flexible manipulator with composite granular jamming and membrane coupling," in Proc. 2012 IEEE/RSJ Int. Conf. Intell. Robots Syst., Oct. 2012, pp. 2922-2927.

[12] E. Brown et al., "Universal robotic gripper based on the jamming of granular material," Proc. Nat. Acad. Sci., vol. 107, no. 44, pp. 18809-18814, 2010.

[13] J. Amend, E. Brown, N. Rodenberg, H. Jaeger, and H. Lipson, "A positive pressure universal gripper based on the jamming of granular material," IEEE Trans. Robot., vol. 28, no. 2, pp. 341-350, Apr. 2012.

[14] S. Hauser, P. Eckert, A. Tuleu, and A. Ijspeert, "Friction and damping of a compliant foot based on granular jamming for legged robots," in Proc. 2016 6th IEEE Int. Conf. Biomed. Robot. Biomechatron., 2016, pp. 1160-1165.

[15] J. M. Bajkowski, B. Dyniewicz, and C. I. Bajer, "Damping properties of a beam with vacuum-packed granular damper," J. Sound Vib., vol. 341, pp. 74-85, Apr. 2015.

[16] E. Steltz, A. Mozeika, N. Rodenberg, E. Brown, and H. Jaeger, "JSEL: Jamming skin enabled locomotion," in Proc. 2009 IEEE/RSJ Int. Conf. Intell. Robots Syst., Oct. 2009, pp. 5672-5677.

[17] A. J. Loeve, O. S. van de Ven, J. G. Vogel, P. Breedveld, and J. Dankelman, "Vacuum packed particles as flexible endoscope guides with controllable rigidity," Granular Matter, vol. 12, no. 6, pp. 543-554, Dec. 2010.

[18] A. Jiang, A. Ataollahi, K. Althoefer, P. Dasgupta, and T. Nanayakkara, "A variable stiffness joint by granular jamming," in ASME 2012 International Design Engineering Technical Conferences and Computers and Information in Engineering Conference. New York, NY, USA: Amer. Soc. Mech. Eng., 2012, pp. 267-275.

[19] S. Rosset, P. Gebbers, B. M. O'Brien, and H. R. Shea, "The need for speed," in SPIE Smart Structures and Materials + Nondestructive Evaluation and Health Monitoring. Bellingham WA, USA: International Society for Optics and Photonics, Apr. 2012, pp. 834004-1-834004-12.

[20] M. A. Robertson, J. M. F. Marin, H. Sadeghi, and J. K. Paik, "Soft pneumatic actuator fascicles for high force and reliability," Soft Robot., Oct. 2016, doi: 10.1089/soro.2016.0029. 